\title{
THE MOST ROBUST CONTROL FACTOR VALUES FOR RECORDING WITH THERMAL INTERFERENCE UNIFORMING TECHNIQUE ON DIRECT OVERWRITE MAGNETO-OPTICAL DISK
}

\author{
K. MORITA, J. SAITO, K. MIYATA, S. KURITA and H. AKASAKA
}

\author{
Nikon Corporation, New Business Development Department. MO Group, 471 Nagaodai-machi, Sakae-ku, \\ Yokohama-shi, Kanagawa 244, Japan
}

\begin{abstract}
We have attempted to obtain the parameters of the recording laser waveform by computer simulation. For the laser waveform, we have used seven kinds of control factors. For the fluctuation of the mark shapes, we have used three kinds of noise factors. To find the optimum control factor values, we have calculated various mark shapes using a computer simulation model which we have already provided for the DOW-MO disk. We have calculated the mark shapes of each combination of control factors under the nine combinations of noise factors. Judging from the controllability of the mark edges, we have obtained the best, that means 'the most robust', control factor values. The best control factor values are eventually the same as the standard values which we have already published in Reference [1].

KEYWORDS: ROBUST, PARAMETER DESIGN, CONTROL FACTOR, NOISE FACTOR, MAGNETO-OPTICAL DISK, DIRECT. OVERWRTE, DOW, THERMAL INTERFERENCE UNIFORMING, TIU
\end{abstract}

\section{INTRODUCTION}

In order to improve the performance of MO disk memory, we assert Direct Overwrite (DOW) for a high data transfer rate, and attempt to raise the recording density with mark edge recording. In mark edge recording where the positions of the leading and the trailing edges have the coded information, it is important to control accurately the shape of the marks. Mark edge recording requires a control to deal with the thermal interference in forming marks. Last year, one of such controls; the thermal interference uniforming (TIU) technique was demonstrated on the DOW-MO disk (in Reference[1]). In this paper, we report the most robust (that means, the most stable to realize an accurate control of the shapes even when fluctuated) control factor values for TIU $0.56 \mu$ $\mathrm{m} / \mathrm{bit}$ recording on the DOW-MO disk.

\section{EXPERIMENT}

\section{Parameter Design (in Reference [2])}

We have used a parameter design method in applying this computer simulation. The parameter design method is one of quality engineering (in alias, TAGUCHI method). The quality engineering has three design steps. The first step is system design. The second is the parameter design. The last is tolerance design. The reason using the parameter design method is to determine the values of parameters. In this case, the parameters are the times and the powers composing write laser waveform.

\section{1-1. Control \& Noise Factors}

For the write laser waveform, we used time parameters(Tw1, Tw2, TL1, TB), and power
parameters(Pw1, Pw2, Pl1, PL2, PL3, PB, PF, PR) as in Figure 1. The position of the leading edge is controlled by $\mathrm{P}_{\mathrm{W}}, \mathrm{P}_{\mathrm{L} 1}$ and Tw1. The width of the mark is controlled by PW2, PL2 and Tw2. The position of the trailing edge is controlled by $\mathrm{PB}_{\mathrm{B}} \mathrm{P}_{\mathrm{F}}$ represents the thermal influence from the front marks. $P_{R}$ represents that of the rear marks. PL1 is bias power heating and $\mathrm{PB}$ is heat shutoff power.

These parameters are fundamental for the TIU technique. Among these parameters, we have selected seven; $\mathrm{Pw}$, PL1, Pw2, PL2, PB, Tw1, and TB as control factors which are shown in Table $1 . \quad$ The control factors are controllable from external systems. Each value of the control factor has three levels. We have adopted the values in Reference[1] as the standard values of level 2 . Thus, the total number of combinations of the control factors could be 3 ?

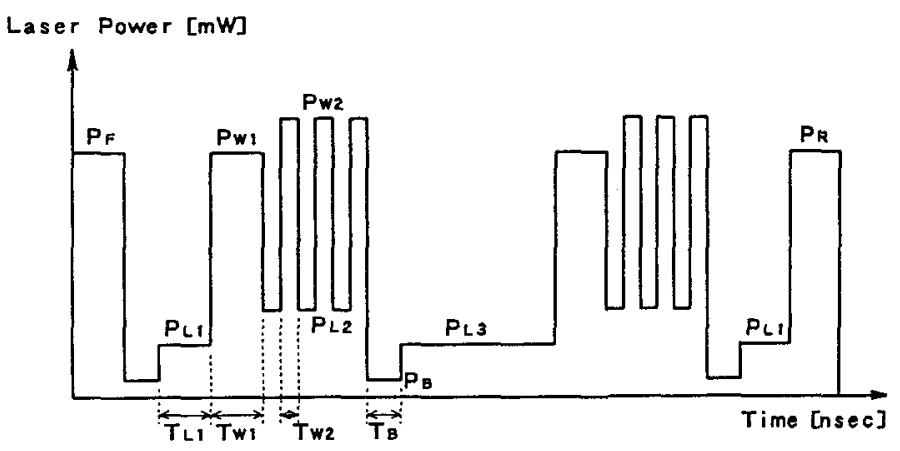

Figure 1. Write Laser Waveform (typical, for 5T mark). 
For fluctuation of the write laser waveform, that means, fluctuation of the mark shapes and their edge positions, we have used the noise factors shown in Table 2. Noise factors are inheritantly uncontrollable. We have selected the parameters $\mathrm{PF}, \mathrm{PR}$, and $\mathrm{PL} 3$ as thermal effects to the objective marks and $\Delta \mathrm{PW}_{2}, \triangle \mathrm{PL}_{1}, \triangle \mathrm{PL}_{\mathrm{L}}, \Delta \mathrm{P}_{\mathrm{L}}, \Delta \mathrm{TW}, \Delta$ $T W 2$, and $\Delta T L 1$ as the fluctuation of the laser waveform. The thermal diffusivity $\kappa$ means the difference of medium thermal characteristics. Each value of the noise factor has three levels like the control factors. The standard values are the same as the level of the control factors. The total number of combinations of noise factors could be $3^{3}$.

The objective performance in this experiment is the linearity of the length of the objective marks. Accordingly, as the signal factors in this experiment, we have chosen three kinds of mark length $2 \mathrm{~T}, 5 \mathrm{~T}$ and $8 \mathrm{~T}$ (that is the shortest, medium and the longest mark length at 1-7 modulation).

\begin{tabular}{|c|c|c|c|c|c|c|c|c|}
\hline & \multicolumn{7}{|c|}{$F a c t a r s$} \\
\hline & & Pw1 & $P_{L}$ & $P_{w 2}$ & $P_{L Z}$ & $P_{g}$ & $T_{w 1}$ & $T_{B}$ \\
\hline Standard $V$ & & 9. $6 \mathrm{mw}$ & 4. $2 \pi m$ & $10.3 \mathrm{mt}$ & 4. $2 \mathrm{mw}$ & b. $75 \mathrm{~mm}$ & $60 \mathrm{n}=$ & $40 n=$ \\
\hline \multirow{3}{*}{ Levels } & 1 & $-10 x$ & $-10 x$ & $-10 x$ & $-10 x$ & $-100 x$ & $-10 x$ & $-10 x$ \\
\hline & 2 & $0 \times$ & $0 x$ & $0 \times$ & ox & $0 x$ & $0 \times$ & $0 x$ \\
\hline & 3 & $+10 x$ & $+10 x$ & $+10 x$ & $+10 x$ & $+100 x$ & $+10 x$ & $+10 x$ \\
\hline
\end{tabular}

Table 1. Control Factors.

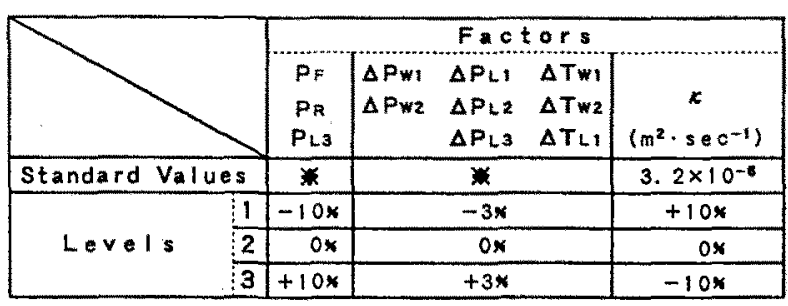

( $\%$ according to control factors)

Table 2. Noise Factors.

\section{1-2. Orthogonal Array}

We have used L18 orthogonal array and L9 orthogonal array shown in Table 3-1 and Table 3-2. With these arrays, we do not have to simulate all combinations of the control factors $\left(3^{7}\right)$, the noise factors $\left(3^{3}\right)$ and the signal factors(3). Assigning the experimental parameters into L18 and L9, we have been able to efficiently examine the whole situation by simulating only $18 \times 9$ kinds of combinations. Each of the 18 combinations of the control factors is fluctuated by 9 combinations of the noise factors.
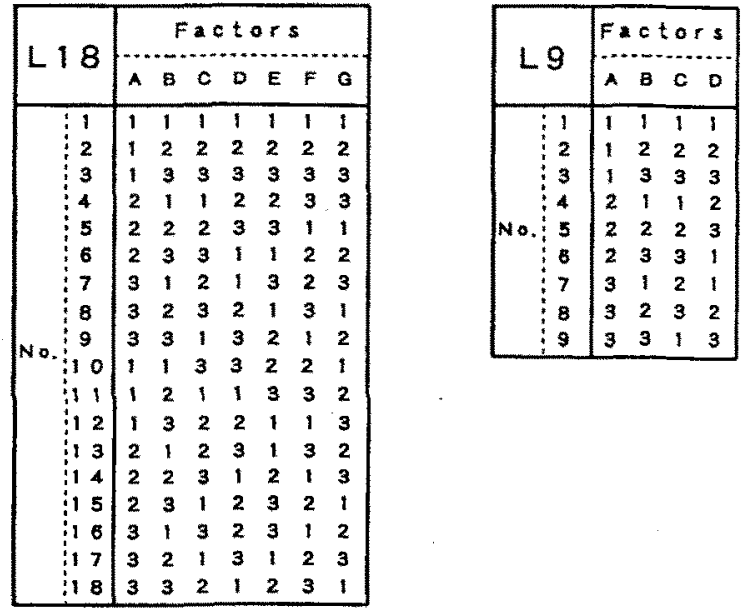

3-1. L18

3-2. L9

Table 3. Orthogonal Array.

In Table 3-1, A G are control factors. In Table 3-2, $A$ is signal factor, B D are noise factors. The numbers 1,2 and 3 are factor levels.

\section{Simulation Model of Thermal Diffusion on DOW-MO} Disk (in Reference [3])

To simplify this simulation, we have modeled the characteristics of DOW-MO disks, and have expressed the model with three main fitting parameters as follows; the specific heat $\mathbf{C} \rho$, the thermal diffusivity $\kappa$, and the adiabatic index $\mathbf{n}$. We have fitted these parameters so as to simulate, with fidelity, the actual properties which we have already measured for the DOW-MO disk. Table 4 shows the fitted result of these parameter values.

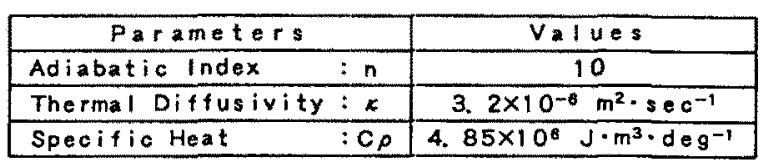

Table 4. Fitting Parameters.

\section{Calculation Method}

Using the above mentioned simulation model, we have calculated the temperature profiles caused by the irradiation with these $18 \times 9$ kinds of write laser waveforms. From the calculated result, the mark shapes appear as in Figure 3. 


\section{RESULTS \& DISCUSSION}

\section{Robustness (in Reference [2])}

In this experiment, the ideal goal is that, even in any fluctuation, the length of mark is in proportion to the duration of laser waveform, and that, even in any fluctuation, the width of mark is constant. Figure 2-1 and 2-2 explain the above. So we can obtain results which analyze the differences between ideal values and simulated values.

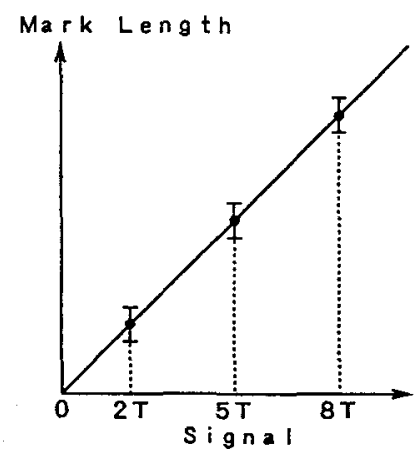

$2-1$.

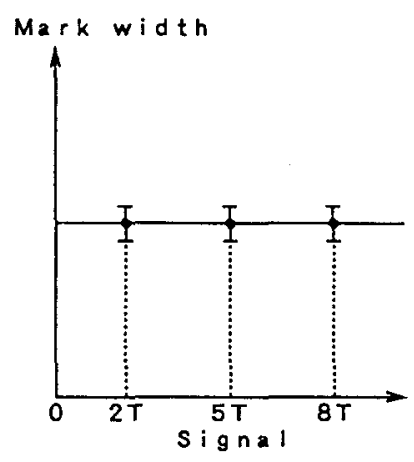

$2-2$.

Figure 2. Ideal Relation.

Figure 2-1 shows relation between Mark Length and Signal, and 2-2 shows relation between Mark Width and Signal.

\section{Result}

\section{2-1. Concerning Mark Length}

The values of Pw1 and Pw2 show the different tendency between the short marks and the long marks. As for the short mark, the better value of PW1 is higher than the standard value of Pw1. The better value of $\mathrm{PW}_{2}$ is lower than the standard value of Fw2. As for the long mark, the better value of Pw1 is lower than the standard value of Pw1. The better value of Pw2 is higher than the standard value of Pw2. The other factor values do not participate in the stability of the mark shapes. Namely, for the values of other factors, the standard values are the better values.

\section{2-2. Concerning Mark Width}

The tendency of results is similar to that of the mark length. As for the short mark, the better value of Pw1 is higher than the standard value of Pw1. The better value of Pw2 is lower than the standard value of Pw2. As for the long mark, the better value of Pw1 is lower than the standard value of Pw1. The better value of Pw2 is higher than the standard value of $\mathrm{P} w 2$. The other factor values do not participate in the stability of the mark shapes.
Namely, for the values of other factors, the standard values are the better values. The reason is similar to that of the mark length.

\begin{tabular}{|c|c|c|c|c|}
\hline & Pwort Mark & high & low & sthers \\
\hline \multirow{2}{*}{ Mark Length } & Long Mark & low & hlgh & std. \\
\hline \multirow{2}{*}{ Mark Width } & Short Mark & high & low & std. \\
\cline { 2 - 6 } & Long Mark & low & high & std. \\
\hline
\end{tabular}

Table 5. Better Control Factors for Short and Long Marks, at Mark length and width.

Judging from the above result, both Pw1 and Pw2 strongly affect for the stability of mark shapes. We have presented this result in Table 5. Considering the controllability of the write laser waveform, it is suitable to choose the moderate values of Pw1 and Pw2. Accordingly, the moderate values are equal to the standard values in Table 1. The best control factor values are eventually the same as the standard values. We have simulated the mark shapes as shown in Figure 3, with the best control factor values under the nine combination of the noise factors. One may observe in Figure 3 that the mark shapes of the best control factors have constant mark width and exhibit controllability of the mark edges, even under the noise factors. In view of these facts, the best control factor values are equivalent to the most robust control factor values.

\section{CONCLUSION}

The authors have obtained the result by computer simulation that the standard values of the write laser waveform which we have adopted (in Reference[1]) is the most robust condition for high density, $0.56 \mu \mathrm{m} / \mathrm{bit}$, mark edge recording on the DOW-MO disk.

\section{REFERENCE}

[1] H.Miyamoto, M.Ojima ,T.Toda, T.Niihara, T.Maeda, J.Saito, H.Matsumoto, T.Hosokawa, and H.Akasaka: Jpn.J.Appl.Phys. Vol.32(1993) pp.5457-5458

[2] G.Taguchi, "Hinshitsu-Kougaku-Kouza 1 Kaihatsu,Sekkeidankai-no-Hinshitsu-Kougaku (Quality Engineering in Development and Design)", Nihon-Kougaku-Kyoukai, Tokyo, 1988 (in Japanese)

[3] J.Saito, K.Morita, S.Kurita, M.Doi, Y.Aoki, and H.Akasaka: Jpn.J.Appl.Phys. Vol.32(1993) pp.51975201 


\begin{tabular}{|l|}
\hline 0 \\
\hline
\end{tabular}
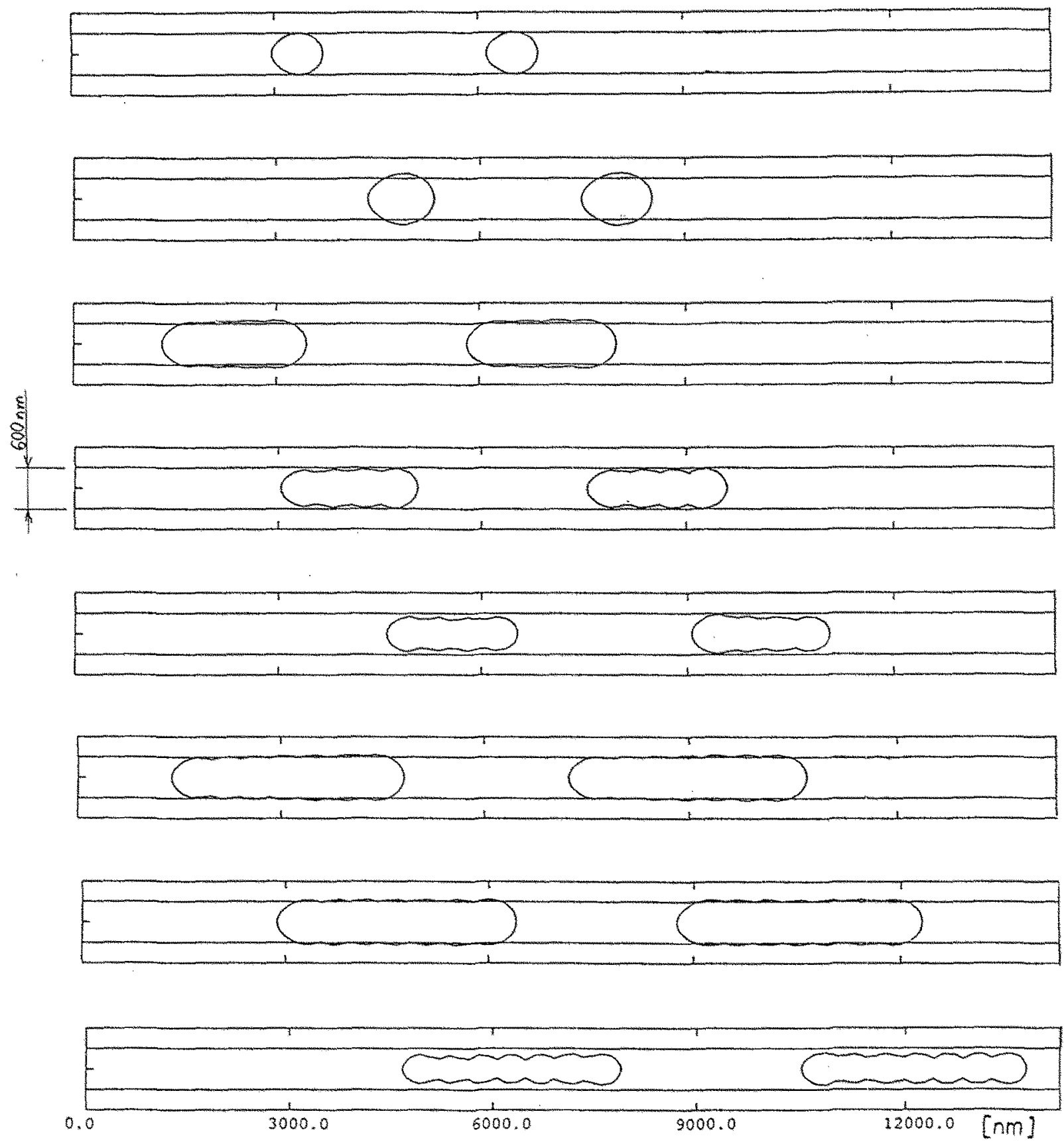

Figure 3. Mark Shapes of The Best Control Facotrs under The Nine Combinations of Noise Factors. Mark length on upper 3 rows is $2 \mathrm{~T}$; that on middle 3 rows is $5 \mathrm{~T}$; and that on lower 3 rows is $8 \mathrm{~T}$. 